ITEP-TH-22/06

\title{
Spontaneous Creation of the Brane World and Direction of the Time Arrow
}

\author{
A.S. GorskY \\ Institute of Theoretical and Experimental Physics \\ B. Cheremushkinskaya ul. 25, 117259 Moscow, Russia
}

\begin{abstract}
In this note we consider the spontaneous creation of the brane world in five-dimensional space with nondynamical external four-form field via spherically asymmetric bounce solution. We argue that spherically asymmetric bounce suggests several inequivalent directions of the time arrow upon the analytic continuation to the space-time with Lorentzian signature. It it shown that S-branes in the imaginary time emerge naturally upon the particular continuation.
\end{abstract}

1. The notion of wave function of the universe [1] is important in the quantum cosmology. It depends on the metric and possible matter fields at $3 \mathrm{~d}$ space slice and can be obtained via the standard quantum mechanical rules via summation over all geometries with particular $3 \mathrm{~d}$ boundary. There are several proposals and prescriptions concerning its actual calculation $[1,2,3]$ however this issue is still under debates since "tunneling from nothing" scenario of Vilenkin and "no-boundary" prescription of Hartle-Hawking yield different exponential factors at the quasiclassical regime. This wave function should obey Wheeler-De Witt equation where the scale factor plays the role of canonical coordinate in the minisuperspace approximation.

This issue can be tested in the brane world scenario [4] implying the localization of the gravity on the brane [5] (see [6] for a review). Similar to the "tunneling from nothing" scenario it is natural to consider the spontaneous creation of the brane world. Being the solitonic object from five-dimensional point of view four-dimensional brane world can be produced nonperturbatively in a higher dimensional generalization of the Schwinger process. The creation of the extended objects in the external flux has been previously used to reduce cosmological constant [7] and was generalized for the case of the multiple fluxes in [8].

The explicit examples of the nonperturbative production of the brane world deal with the Schwinger type process in the external nondynamical four-form field in five dimensions $[9,10]$ or with the production of the bubble of the true vacuum in the false vacuum in the theory with additional scalar field and peculiar potential [11]. The wave function of the universe can be treated in this way more literally since it corresponds to the wave function of the brane degrees of freedom [12] and metric on the brane is fixed classically by the geometry of embedding.

It shall be important in what follows that bounces found in $[9,10]$ are spherically asymmetric and have several turning points. Recently gravitational spherically asymmetric bounces were considered as well $[13,14,15]$. The asymmetry of gravitational bounces emerges if the quantum 
radiation during tunneling or before tunneling is taken into account. It turns out that in this case the emerging wave function of the universe depending on the scale factor predicts the finite nonvanishing value of the cosmological constant.

To define the wave function of the universe one has to select the direction of the time arrow. In the spontaneous creation of universe via tunneling process there is no initial prescription of the time arrow which is fixed by the surface where continuation from the Euclidean to the Lorentzian geometries takes place. The general conditions imposed on these surfaces have been discussed in [16].

In this note we shall discuss the inequivalent continuations into the Lorentzian space-time of the spherically asymmetric Euclidean bounce solution in the external four-form field in five dimensions yielding the spontaneous creation of the brane world. Our solution involves simultaneously charged and neutral branes. It turns out that spherically asymmetric bounces allow several inequivalent continuations with different brane content upon the materialization. That is spherically asymmetric Euclidean solution amounts to several wave functions of the universe or directions of the time arrow. One choice of the continuation surface amounts to the multiple neutral branes and single charged brane upon the materialization and time arrow turns out to be parallel to the worldvolume of the neutral branes which are at rest in the external field. This type of continuation resembles the process of the particle creation during the tunneling [17] however in our model example the branes created are involved into the asymmetric bounce solution and strongly deform its shape.

Another continuation surface yields only spherical charged brane in the final state however the corresponding wave function of the brane universe involves the summation over the array of the neutral branes. This continuation surface provides the different direction of the time arrow and neutral branes are identified with the S-branes introduced in [18]. Such array of S-branes in imaginary time has been related with the stringy mode of the decaying brane in $[19,21,20]$ that is spontaneous creation of the brane world can be identified with some decaying neutral brane. The brane world is now defined on the charged branes with four-dimensional De Sitter worldvolume geometry.

Note that the possibility of two different time directions induced by continuation of the twodimensional Euclidean solution has been mentioned in [22] where the derivation of the HartleHawking wave function in the framework of topological strings has been discussed. It was applied there to explanation of the relation between the entropy of the black holes and partition functions of the topological strings and was treated as some version of open-closed duality. More recently similar issue has been addressed in $\mathrm{D}=4$ context in [23].

This paper is organized as follows. First we shall describe two examples of the spherically asymmetric bounces responsible for the Schwinger type processes. Then we discuss continuation surfaces corresponding to the different wave functions of the universe and emphasize the essential role of S-branes. We conclude with some speculations and open questions.

2. To start with let us discuss spherically asymmetric Euclidean bounce relevant for the Schwinger pair production in two dimensions. In two dimensional electrodynamics the gauge field is nondynamical however it provides the nonperturbative pair production. The leading contribution to the probability follows from the Euclidean bounce solution which is just circle in two dimensional Euclidean space and represents the worldline of the electron-positron pair. The probability of the process at the exponential accuracy reads as

$$
w_{0} \propto \exp \left(-\frac{\pi m^{2}}{e E}\right)
$$




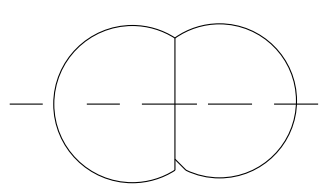

A

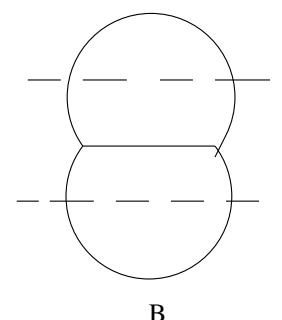

B

Figure 1: Two different orientations of the spherically asymmetric Euclidean bounce responsible for the Schwinger pair production in two dimensions.

where $m$ is the particle mass and $E$ is electric field. However one can consider more complicated asymmetric bounce solutions involving neutral bound states. The examples of such bounce are shown at Fig.1. It is important to emphasize that in nonperturbative pair production process the direction of the time arrow is fixed from the very beginning and the continuation surface from the Euclidean to Lorentzian space-time should be transverse to this fixed direction. That is orientation of the spherically asymmetric bounce in the Euclidean space matters.

As the basic example we consider two different orientations of the same bounce at Fig.1 which result in the different final states upon materialization. The action calculated on this bounce configuration reads as [9]

$$
S=\frac{2 m^{2}}{e E}\left(\pi-\arcsin \left(1-\frac{M^{2}}{4 m^{2}}\right)^{1 / 2}\right)+\frac{2 M m}{e E}\left(1-\frac{M^{2}}{4 m^{2}}\right)^{1 / 2}
$$

where $m(M)$ is the mass of the charged(neutral) particle.

With the continuation surface at Fig.1b we get electron-positron pair in the final state that is asymmetric bounce amounts to the nonperturbative correction to the contribution from the spherically symmetric bounce. The neutral state corresponds to the S-string localized in the Euclidean time. One can easily consider more complicated bounce involving many S-strings that is generic probability of the pair production at the exponential accuracy reads as

$$
W=w_{0}+w_{1}+w_{2}+\ldots
$$

If the mass of the bound state is close to $2 m$ the probability involving $n$ S-strings can be approximated by $\exp \left(-n S_{0}\right)$ and summation over the arbitrary number of S-strings provides the result indicating a kind of thermal behavior

$$
W_{\infty} \propto \frac{e^{-S_{0}}}{1-e^{-S_{0}}}
$$

The effective "temperature" of $(0+1)$ dimensional universe coincides with the Unruh-like temperature for the particle moving with constant acceleration $T=\frac{a}{2 \pi}=\frac{e E}{2 m \pi}$ in the external electric field.

With the different continuation surface at Fig.1a we get charged pair and neutral bound state upon materialization that is different final state which does not exist for the spherically symmetric bounce at all. The bubble involving multiple neutral states presented at Fig.2 yields with the continuation cut A the charged $e^{+} e^{-}$pair and multiple neutral bound states in the final state. Note that although the asymmetric bounce in the Schwinger pair production and the one 


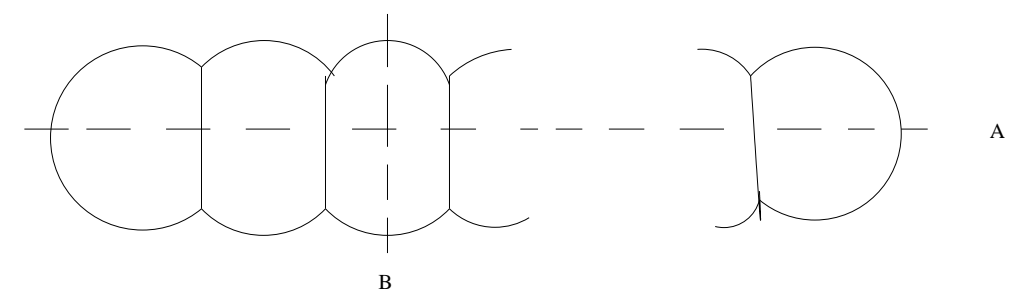

Figure 2: Euclidean bounce involving the large number of neutral branes.

responsible for the creation of the brane world look similar there is important difference. In the Schwinger production the time direction is fixed while in the brane world creation time is defined upon the continuation surface is chosen.

More general situation concerns two simultaneous cuts at Fig.1b and has the different interpretation. With two cuts we get function depending on two radii $\rho\left(R_{1}, R_{2}\right)$ which can be naturally treated as the density matrix similar to case of asymmetric gravitational bounce in [15]. The example represented at Fig.1b corresponds to the density matrix $\rho(R, R)$ since both arguments correspond to the same turning point. In the limit $M=2 m$ the density matrix reduces to the pure state case since the bounce degenerates to two copies of the spherically symmetric bounce. One could also glue bounce along two cuts or two bounces to get the bounce with toroidal geometry corresponding to the thermal behaviour.

3. Let us briefly review spherically asymmetric Euclidean bounce solutions providing the spontaneous creation of the brane world found in $[9,10]$. The simplest model [9] involves asymptotically flat five-dimensional Euclidean space. It is assumed that negative cosmological constant $\Lambda$ is exactly compensated by the positive energy density from the field strength $H=d B$ of the four-form field $\mathrm{B}$ which is nondynamical in five dimensions

$$
\Lambda+\frac{k h_{+}^{2}}{2}=0
$$

where $k$ is five-dimensional gravitational constant and $h_{+}$is the asymptotic value of the Hodge dual of $H$. We assume the existence of 3-branes charged with respect to the external four-form field which can be glued together along some junction manifolds.

The bounce configuration is the generalization of spherically symmetric bounce responsible for the Schwinger type production of branes discussed in [7]. The asymmetric bounce involves two spherical segments of the charged branes glued along the circle junction with the neutral brane with the disc topology which is higher dimensional analogue of Fig.1. The external field jumps across the charged branes and yields the effective cosmological constant inside the bounce

$$
\Lambda_{\text {eff }}=\frac{k\left(h_{-}^{2}-h_{+}^{2}\right)}{2}
$$

with the corresponding radius of $A d S_{5}$ geometry

$$
R_{A d S_{5}}^{2}=-\frac{24}{\Lambda_{e f f}}
$$

The metric to the right of the neutral brane inside the bubble reads as

$$
d s^{2}=\frac{d z^{2}+d \rho^{2}+\rho^{2} d \Omega_{3}^{2}}{\left(1-\frac{(z-a)^{2}+\rho^{2}}{R_{a d s}^{2}}\right)^{2}}
$$


while the metric to the left of the neutral brane inside the bubble is

$$
d s^{2}=\frac{d z^{2}+d \rho^{2}+\rho^{2} d \Omega_{3}^{2}}{\left(1-\frac{(z+a)^{2}+\rho^{2}}{R_{a d s}^{2}}\right)^{2}}
$$

The parameter $a$ can be determined from the Israel condition and equals to

$$
a=\frac{4 T_{0}}{h_{+}^{2}-h_{-}^{2}}
$$

where $T_{0}$ is the tension of the neutral brane. The brane world on the neutral brane enjoys $A d S_{4}$ geometry.

More general bounce in the $A d S_{5}$ geometry has been found in [10]. In this case one considers three charged branes glued together along junction manifold. The branes are considered now as test objects that it their back reaction on the metric is neglected. This solution is based essentially on the consideration of the minimal charged surfaces in AdS with the homogeneous field described in [24]. They are classified into three classes: undercharged ones (saturating a sort of BPS inequality between charge and tension of the brane), overcharged ones (breaking the BPS inequality) and BPS ones. Our bounce now is glued out of three pieces of branes - of BPS one, of undercharged one and of overcharged one.

Consider the metric of AdS as in [24],

$$
d s^{2}=R_{a d s}^{2}\left(\cosh ^{2} \rho d \tau^{2}+d \rho^{2}+\sinh ^{2} \rho d \Omega_{d-2}^{2}\right),
$$

where $R_{a d s}$ is the anti-de Sitter radius, and assume that the curvature form $H=d B$ of the $B$-field is proportional to the volume form with a constant coefficient (flux density). Assuming the spherical symmetry of the brane worldsheet, one reduces the effective action to :

$$
S=T R_{a d s}^{d-1} \Omega_{d-2} \int d \tau\left[\sinh ^{d-2} \rho \sqrt{\cosh ^{2} \rho+\left(\frac{d \rho}{d \tau}\right)^{2}}-q \sinh ^{d-1} \rho\right]
$$

where $\Omega_{d-2}=\frac{2 \pi \frac{(d-1)}{2}}{\Gamma\left(\frac{d-1}{2}\right)}$ stands for the volume of a unit $(d-2)$ sphere and $q$ is a constant made out of the flux density, brane charge and the brane tension. The condition $q=1$ is identified in [24] as the BPS one. The branes with $q<1$ will be referred to as undercharged ones and those with $q>1$ - as overcharged.

Upon the change of variables,

$$
\tanh \tau=\tan \theta
$$

the metric (11) is put to the form

$$
d s^{2}=R_{a d s}^{2} \frac{d \tau^{2}+d \theta^{2}+\sin ^{2} \theta d \Omega_{d-2}^{2}}{\cos ^{2} \theta}
$$

The minimal surfaces for the case $q<1$ look as follows:

$$
\cosh \rho=\frac{\sinh \tau_{m}}{\sinh \left(\tau+\tau_{0}\right)}
$$


where $\tanh \tau_{m}=q$.

In coordinates (13) the undercharged surfaces take the form

$$
\cos \theta=\frac{\sinh \left(\tau+\tau_{0}\right)}{\sinh \tau_{m}} .
$$

Restriction of the $A d S_{5}$ metric (14) onto the undercharged surfaces (18) gives the metric of $A d S_{4}$. In the case $q>1$ the relevant charged minimal surfaces look as follows:

$$
\cosh \rho=\frac{\cosh \rho_{m}}{\cosh \left(\tau+\tau_{0}\right)}
$$

where tanh $\rho_{m}=1 / q$. In coordinates (13) the overcharged surfaces take the form

$$
\cos \theta=\frac{\cosh \left(\tau+\tau_{0}\right)}{\cosh \rho_{m}} .
$$

Restriction of the $A d S_{5}$ metric (14) onto the overcharged surfaces (18) gives the metric of the sphere $S_{4}$. The BPS case $(\mathrm{q}=1)$ can be obtained as a limit from either of the cases above. Corresponding surfaces look as follows :

$$
\cos \theta=\frac{1}{z_{0}} e^{\tau}
$$

where $z_{0}$ is a constant. Apparently, restriction of the AdS metric onto these surfaces gives flat Euclidean metric.

The bounce which describes the tunneling into the brane world [10] is glued out of three pieces - a piece of BPS brane located along $z=z_{0}$ section, where the brane world with the flat metric is defined, a piece of undercharged brane, (16), located above the BPS brane and playing a role of one regulator brane, and a piece of overcharged brane, located below the BPS brane and playing the role of the other regulator brane. All three pieces are glued along the junction manifold. The usual junction conditions are the charge conservation and the tension forces balance. Apparently, the configuration sketched above has a finite action since none of the constituting pieces reaches the AdS boundary, hence the tunneling goes with a finite probability which can be easily computed.

4. To discuss the different possible continuation surfaces let us comment on their generic properties discussed in [16]. It was shown that to have so called real geometries one has to impose the condition of the vanishing external curvature $K=0$ on the continuation surface and the vanishing canonical momenta of all fields involved. In the case of spontaneous creation of the brane world the situation is simpler since we can consider tunneling of the single brane degree of freedom that is radius of the bubble quasiclassically. On the other hand gravity can be treated classically and the emergent geometry on the brane worldvolume is defined by its embedding. That is condition on the continuation surface in our example is vanishing of the canonical momenta $\dot{R}=0$.

Consider the symmetric bounce corresponding to the spontaneous brane world production in the external field. The solution involves spherical bubble formed by the charged brane expanding in the Minkowski space after the turning point. The effective Lagrangian describing the tunneling process depends on the radius of the bubble and reads as

$$
L=-4 \pi T R^{2} \sqrt{1-\dot{R}^{2}}+\frac{4 \pi e H R^{3}}{3}
$$


where $T$ is tension of the charged brane, $H$ is the constant curvature of the external higher-form field and $e$ is the brane charge. The canonical Hamiltonian can be easily derived

$$
H=\sqrt{p^{2}+\left(4 \pi T R^{2}\right)^{2}}-\frac{4 \pi e H R^{3}}{3}
$$

where $p$ is the canonical momentum. The wave function of the universe on the brane $\Psi(R)$ depends on the radius of the bubble and obeys the equation

$$
\hat{H} \Psi(R)=0
$$

The probability of the creation of the bubble can be easily calculated

$$
w \propto \exp \left(-\frac{9 \pi^{2} T^{3}}{g^{2} H^{2}}\right)
$$

It can be assumed that the space outside the bubble is flat but is $A d S_{5}$ inside the bubble. In this simple case all directions of time arrow are equivalent due to the spherical symmetry. The cut of the bubble which yields the direction of time arrow can be identified with any section involving the center of the bubble.

However in more complicated case of spherically asymmetric bounces described above generically there are several possibilities to perform the analytic continuation to the Lorentzian space. Consider the solution to the equation of motion found in [9]. At Fig.1a the simplest analytic continuation is presented with the vertical direction of the Lorentzian time. The process corresponding to this solution looks as follows. First, two small bubbles are produced in the Euclidean space then these bubbles "collide" and form a joint boundary in the Euclidean region. Later on at the turning point the configuration is continued into the Lorentzian space. The action calculated on the solution yields the probability of the process

$$
\text { nothing } \rightarrow \text { charged brane }+ \text { neutral brane }
$$

The configuration arising in the Lorentzian space looks like two spherical segments of the charged branes connected by the flat neutral brane. It is assumed that brane world is defined on the neutral brane. The neutral brane has $A d S_{4}$ worldvolume metric and charged brane provides a kind of regularization.

Another continuation into the Lorentzian space is possible with the vertical direction of time at Fig 1b. In this case we fix initial and final states in the process to be

$$
\text { nothing } \rightarrow \text { charged brane. }
$$

and asymmetric bounce provides the nonperturbative correction to the probability defined by spherically symmetric one. That is the total probability from two bounces reads as $w_{0}+w_{1}$ where $w_{0}$ is the contribution prom the spherically symmetric bounce and $w_{1}$ is correction amounted from Fig.1b type configuration involving S-brane localized at the imaginary time.

Consider more general asymmetric bounce involving multiple branes. The example of the corresponding configuration is presented at Fig.2 and the metric between two neighbor branes reads as

$$
d s^{2}=\frac{d z^{2}+d \rho^{2}+\rho^{2} d \Omega_{3}^{2}}{\left(1-\frac{\left(z-a_{n}\right)^{2}+\rho^{2}}{R_{a d s}^{2}}\right)^{2}}
$$


where the position of the center of $n$-th neutral brane equals to

$$
a_{n}=(2 n-1) a
$$

With the continuation surface $A$ we get charged brane with large number of neutral branes in the final state while the continuation surface $B$ amounts to the charged brane only. Evidently the contribution of this solution to the tunneling probability is suppressed compared to the single intermediate brane case. Let us also note that since S-branes have wrong kinetic terms corresponding to the fluctuations in the time direction with the infinite number of S-branes we have a kind of dynamical mechanism supporting the "deconstruction of time" scenario [26].

Note the interpretation of S-branes in the imaginary time developed in [19, 20, 21]. According to this interpretation the infinite chain of S-branes can be related to the closed string mode of the decaying brane located at the fixed time. It has been proven in the stringy framework that the background of the S-branes in the imaginary time is equivalent to the additional boundary state on the worldsheet. Equivalently one could introduce the tachyon potential

$$
\delta L=\lambda \cosh X_{0}
$$

where $\lambda$ fixes the distance between the S-branes in the imaginary time direction

$$
\lambda \propto \delta t_{E}
$$

and the lifetime of the decaying brane. The analysis of [19] implies that the relation with the closed string modes survives for the finite number of S-branes distributed irregularly in the imaginary time. The value of $\lambda=1 / 2$ corresponds to the special case when $\mathrm{S}$-branes provide the Dirichlet boundary conditions for the open strings.

Comparing this picture with our model case we conclude that array of the neutral S-branes in the imaginary time can be identified with the decaying closed string. Note that to justify the quasiclassical calculation we have to assume that the radii of the corresponding bubbles are large enough. Hence it is naturally to expect that boundary effects on the neutral S-branes don't change this interpretation. Let us remark that idea to use decaying brane for the generation of matter in the cosmological framework has been discussed in [25].

The wave function of the universe depends on the size of the continuation surface which simultaneously provides the quasiclassical metrics on the worldvolume of the brane. If we consider continuation surface of the type B at Fig 2. the total wave function of the universe of this type looks as

$$
\Psi_{\infty}=\sum_{k=0}^{\infty} a_{k} \psi_{k}
$$

where $\psi_{k}$ corresponds to the wave function with $k$ S-branes in the imaginary time involved. The total probability of the quantum creation of the brane world in this channel is related to $\Psi_{\infty}$ as follows

$$
e^{-S} \propto<\Psi_{\infty} \mid \Psi_{\infty}>
$$

and different terms in the scalar product correspond to the different continuation surfaces into the Lorentzian space-time. For instance, two different horizontal cuts at Fig.1b correspond to the contributions $\left\langle\Psi_{0}\right| \Psi_{1}>$ and $\left\langle\Psi_{1}\right| \Psi_{0}>$ to the probability of the process. Just as in twodimensional toy example if we make two horizontal cuts simultaneously the emerging object can be identified with the density matrix of the brane universe. 

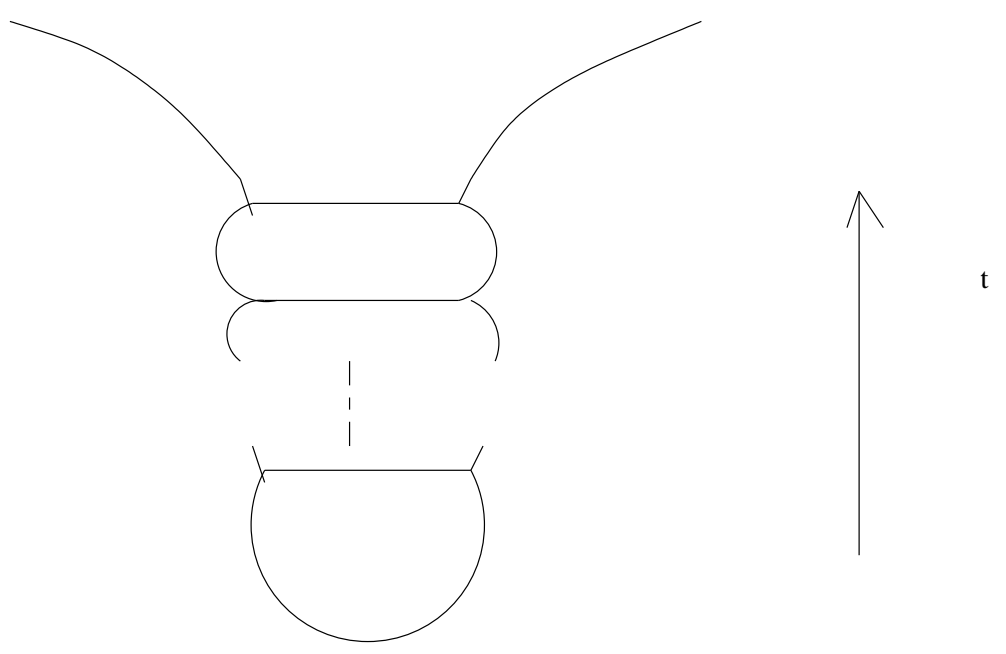

Figure 3: The schematic representation of the contribution to the total Hartle-Hawking wave function $\Psi_{\infty}$ involving the array of S-branes in the imaginary time.

For the continuation surfaces of the type A at Fig.2 the total wave function of the universe can be defined as the infinite sum as well

$$
\Phi_{\infty}=\sum_{k=0}^{\infty} b_{k} \phi_{k}
$$

however with the different interpretation. If the brane world is defined on the charged brane then $\phi_{k}$ corresponds to the contribution to the wave function involving $\mathrm{k}$ additional neutral branes. Contrary if the brane world is defined on stack of the neutral branes then charged branes play the role of additional matter. Note that we have the simple relation

$$
<\Psi_{\infty}\left|\Psi_{\infty}>=<\Phi_{\infty}\right| \Phi_{\infty}>
$$

whose counterpart exists for each particular asymmetric bounce.

The similar analysis can be done for the spherically asymmetric gravitational bounce discussed in $[13,14,15]$. In this case there are several inequivalent continuation surfaces as well which differ by the different values of the scale factors in the emerging universes.

5. In this note we have argued that generically spherically asymmetric bounce describing nucleation of the brane world admits several inequivalent continuations to the Lorentzian space-time with the different directions of the time arrow and Hartle-Hawking wave functions corespondingly. Contrary to the asymmetric gravitational bounce in [15] we treat "matter" quasiclassically implying the classical gravity. We analyzed the simplest example of nucleation of the brane world in the nondynamical four-form field with the constant curvature. The existence of the neutral and charged branes with respect to the external field was assumed in our model example. In this case there are two essentially different continuations into the Lorentzian signature space. One continuation yields brane world on the expanding charged brane with the positive cosmological constant. The second continuation provides the brane world on the neutral brane or array of neutral branes at rest with negative cosmological constant. To some extent the time variable in one continuation can be identified with the RG scale in another one.

The natural question concerns the most preferable final state of the nucleation process. It seems that it is natural to consider all possible continuation surfaces on the equal footing hence 
one has to sum over brane worlds emerged. Probably some entropy factors could be essential due to the different number of branes in the final state .

Another point of concern deals with the multiple asymmetric bounces. In the case of spherically symmetric Euclidean bounces summation over the number of bounces does not amount to the selection problem for the direction of the time arrow. However in asymmetric case the situation is more subtle. Consider for example the Euclidean solution involving two asymmetric bounces with different orientations of the neutral branes. It is impossible to consider the same continuation surface for them since they have different turning points. Hence one could consider more complicated continuation surface in the Euclidean space or take into account the interaction between the bounces. The interaction of the differently oriented bounces seems to be minimized when neutral branes are parallel however this point needs for the separate analysis.

In this note we considered the bounce built from the set of branes with the same dimension, say 3-branes providing four-dimensional brane world. However the bounces with the different brane content could be considered as well. For instance, we can consider the four-rank field creating the 3-brane spherically asymmetric bubble with the set of strings inside. In this case in one "continuation channel" we get four dimensional brane world on 3-brane worldvolume. In the different "channel" we get single 3-brane and set of strings. Oppositely one can consider higher rank form fields creating 5 -branes. The asymmetric bounce could be obtained from the bubble of 5 -brane with the number of 3-branes inside. Then in one channel we can consider the creation of five-dimensional brane world while in the second channel the creation of the brane world on 5-brane or 3-branes.

Finally let us note that the opposite process of the brane world decay in the external field is possible. To this aim consider the neutral 3-brane in the external four-four field with the constant curvature. The decay can occur due to the Euclidean bounce configuration corresponding to the induced Schwinger process in higher dimensions similar to the processes considered in [27]. It can be considered as an example of the generic Myers phenomena in the external fields [28]. From the worldvolume point of view the process of decay looks as the blow up of the four dimensional brane world into the fifth dimension.

I am grateful to A. Barvisnky and A.Rosly for the useful comments. The work was supported in part by grants CRDF RUP2-261-MO-04 and RFBR-04-011-00646.

\section{References}

[1] J. B. Hartle and S. W. Hawking, "Wave Function Of The Universe," Phys. Rev. D 28, 2960 (1983).

[2] A. Vilenkin, "Creation Of Universes From Nothing," Phys. Lett. B 117, 25 (1982).

A. Vilenkin, "The Birth Of Inflationary Universes," Phys. Rev. D 27, 2848 (1983).

[3] A. D. Linde, "Quantum Creation Of The Inflationary Universe," Lett. Nuovo Cim. 39, 401 (1984).

[4] V. A. Rubakov and M. E. Shaposhnikov, "Do We Live Inside A Domain Wall?," Phys. Lett. B 125 (1983) 136.

K. Akama, "An Early Proposal Of 'Brane World'," Lect. Notes Phys. 176, 267 (1982). 
[5] L. Randall and R. Sundrum, "An alternative to compactification," Phys. Rev. Lett. 83, 4690 (1999) [arXiv:hep-th/9906064].

[6] V. A. Rubakov, "Large and infinite extra dimensions: An introduction," Phys. Usp. 44, 871 (2001) [Usp. Fiz. Nauk 171, 913 (2001)] [arXiv:hep-ph/0104152].

[7] J. D. Brown and C. Teitelboim, "Neutralization Of The Cosmological Constant By Membrane Creation," Nucl. Phys. B 297, 787 (1988).

[8] R. Bousso and J. Polchinski, "Quantization of four-form fluxes and dynamical neutralization of the cosmological constant," JHEP 0006, 006 (2000) [arXiv:hep-th/0004134].

[9] A. Gorsky and K. Selivanov, "Tunneling into the Randall-Sundrum brane world," Phys. Lett. B 485, 271 (2000) [arXiv:hep-th/0005066].

[10] A. S. Gorsky and K. G. Selivanov, "Big bang in $\operatorname{AdS}(5)$ with external field and flat 4d universe," Int. J. Mod. Phys. 16, 2243 (2001) [arXiv:hep-th/0006044].

[11] J. Garriga and M. Sasaki, "Brane-world creation and black holes," Phys. Rev. D 62, 043523 (2000) [arXiv:hep-th/9912118].

[12] R. Cordero and A. Vilenkin, "Stealth branes," Phys. Rev. D 65, 083519 (2002) [arXiv:hepth/0107175].

[13] S. Sarangi and S. H. Tye, "A note on the quantum creation of universes," arXiv:hepth/0603237.

H. Firouzjahi, S. Sarangi and S. H. H. Tye, "Spontaneous creation of inflationary universes and the cosmic landscape," JHEP 0409, 060 (2004) [arXiv:hep-th/0406107].

[14] R. Brustein and S. P. de Alwis, "The landscape of string theory and the wave function of the universe," Phys. Rev. D 73, 046009 (2006) [arXiv:hep-th/0511093].

[15] A. O. Barvinsky and A. Y. Kamenshchik, "Cosmological landscape from nothing: Some like it hot," arXiv:hep-th/0605132.

[16] G. W. Gibbons and J. B. Hartle, "Real Tunneling Geometries And The Large Scale Topology Of The Universe," Phys. Rev. D 42, 2458 (1990).

J. J. Halliwell and J. B. Hartle, "Integration Contours For The No Boundary Wave Function Of The Universe," Phys. Rev. D 41, 1815 (1990).

[17] V. A. Rubakov, "Quantum Mechanics In The Tunneling Universe," Phys. Lett. B 148 (1984) 280 .

[18] M. Gutperle and A. Strominger, "Spacelike branes," JHEP 0204, 018 (2002) [arXiv:hepth/0202210].

[19] D. Gaiotto, N. Itzhaki and L. Rastelli, "Closed strings as imaginary D-branes," Nucl. Phys. B 688, 70 (2004) [arXiv:hep-th/0304192].

[20] N. Lambert, H. Liu and J. Maldacena, "Closed strings from decaying D-branes," arXiv:hepth/0303139. 
[21] A. Maloney, A. Strominger and X. Yin, "S-brane thermodynamics," JHEP 0310, 048 (2003) [arXiv:hep-th/0302146].

[22] H. Ooguri, C. Vafa and E. P. Verlinde, "Hartle-Hawking wave-function for flux compactifications," Lett. Math. Phys. 74 (2005) 311 [arXiv:hep-th/0502211].

[23] B. McInnes, "The geometry of the entropic principle and the shape of the universe," arXiv:hep-th/0604150.

[24] J. M. Maldacena, J. Michelson and A. Strominger, "Anti-de Sitter fragmentation," JHEP 9902, 011 (1999) [arXiv:hep-th/9812073].

[25] S. Kawai, E. Keski-Vakkuri, R. G. Leigh and S. Nowling, "Brane decay from the origin of time," Phys. Rev. Lett. 96, 031301 (2006) [arXiv:hep-th/0507163].

[26] Z. Berezhiani, A. Gorsky and I. I. Kogan, "On the deconstruction of time," JETP Lett. 75, 530 (2002) [Pisma Zh. Eksp. Teor. Fiz. 75, 646 (2002)] [arXiv:hep-th/0203016].

[27] A. Gorsky and K. Selivanov, "Junctions and the fate of branes in external fields," Nucl. Phys. B 571, 120 (2000) [arXiv:hep-th/9904041].

[28] R. C. Myers, "Dielectric-branes," JHEP 9912, 022 (1999) [arXiv:hep-th/9910053]. 